\title{
Carga de trabalho da equipe de enfermagem neonatal e sua relação com características da clientela
}

\author{
Neonatal nursing team workload and its relationship with patient characteristics \\ Carga de trabajo del equipo de enfermería neonatal y su relación con las características del cliente
}

\author{
Aline Patrícia Vicente Franco' $\odot$, Beatriz Pera de Almeida Hamasaki' @ , Luciana Renata de Puiz' @ ,

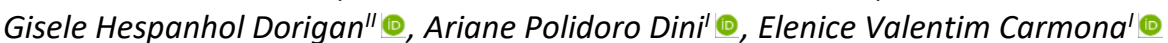

'Universidade Estadual de Campinas, Campinas, São Paulo, Brasil; "Fundação Hermínio Ometto (FHO), Araras, São Paulo, Brasil

\begin{abstract}
RESUMO
Objetivo: avaliar a carga de trabalho de enfermagem em uma unidade de internação neonatal e correlacionar com características clínicas dos pacientes. Método: estudo descritivo e transversal, com o Nursing Activities Score em uma unidade neonatal de hospital público de ensino. Foram incluídos pacientes hospitalizados por no mínimo 24 horas, no período de 60 dias. Os dados foram analisados segundo estatística descritiva e inferencial. Resultados: a amostra foi composta por 115 pacientes, com total de 1944 medidas. A média do Nursing Activities Score foi de 73\%, obtendo-se média de $74 \%$ em leitos de cuidados intensivos e $64 \%$ em cuidados semi-intensivos. Conclusão: identificou-se uma alta carga de trabalho, com média de 17,5 horas de assistência a cada recém-nascido nas 24 horas, o que pode ter impacto na segurança da assistência. Peso de nascimento e idade gestacional podem representar incremento à carga de trabalho dos profissionais.
\end{abstract}

Descritores: Enfermagem Neonatal; Recém-Nascido; Recursos humanos de enfermagem; Carga de Trabalho.

\section{ABSTRACT}

Objective: to evaluate the nursing workload at a neonatal unit and to correlate it to patient clinical characteristics. Method: this descriptive, cross-sectional study applied the Nursing Activities Score to assess nursing workload at a neonatal unit of a public teaching hospital. Patients included were those hospitalized for at least 24 hours over 60-day period. Data were analyzed using descriptive and inferential statistics. Results: the sample comprised 115 patients, yielding a total of 1944 measurements. The average Nursing Activities Score at the unit was $73 \%$, an average of $74 \%$ for intensive care beds and $64 \%$ for semi-intensive ones. Conclusion: high workload was identified at the unit, averaging 17.5 hours of care every 24 hours for each newborn, which can impact the safety of care. Birth weight and gestational age may entail increased workload for nursing staff.

Descriptors: Neonatal Nursing; Infant, Newborn; Nursing Staff; Workload.

\section{RESUMEN}

Objetivo: evaluar la carga de trabajo de enfermería en una unidad de hospitalización neonatal y relacionarla con las características clínicas de los pacientes. Método: se trata de un estudio descriptivo y transversal, utilizando el Nursing Activities Score en una unidad neonatal, en un hospital universitario público. Se incluyeron pacientes hospitalizados durante al menos 24 horas, dentro del período de 60 días. Se analizaron los datos según estadística descriptiva e inferencial. Resultados: la muestra se compuso de 115 pacientes, totalizando 1944 mediciones. El puntaje promedio del Nursing Activities Score fue del 73\%, siendo el promedio para la unidad de cuidados intensivos del $74 \%$ y para los cuidados semi intensivos del $64 \%$. Conclusión: se identificó una alta carga de trabajo en la unidad, con un promedio de 17.5 horas de atención a cada neonato en 24 horas, lo que puede impactar la seguridad de la atención. El peso al nacer y la edad gestacional pueden representar un aumento en la carga de trabajo de los profesionales.

Descriptores: Enfermería Neonatal; Recién Nacido; Personal de Enfermería; Carga de Trabajo.

\section{INTRODUÇÃO}

Em unidades neonatais, destaca-se a atuação dos profissionais de enfermagem no manejo de tecnologias diversas, na necessidade do domínio científico, de experiências e saberes que se configuram para prover assistência peculiar e de alta complexidade no processo de cuidar de prematuros ou bebês a termo que demandam hospitalização, bem como de sua família ${ }^{1}$.

Diante das inovações tecnológicas, enfermeiros têm incorporado em atividades gerenciais o uso de ferramentas para mensurar os riscos e a complexidade assistencial dos pacientes, com o intuito de identificar necessidades e promover a segurança do paciente ${ }^{2}$. Dentre os instrumentos gerenciais disponíveis para avaliar a carga de trabalho da equipe de enfermagem está o Nursing Activities Score (NAS) ${ }^{3}$, traduzido e validado para o Brasil ${ }^{4}$. 
O NAS está dividido em sete categorias de intervenções com 23 itens, cuja pontuação varia de 1,2 a 32 pontos. A pontuação final obtida por meio da somatória de seus itens representa a quantidade de tempo de trabalho de enfermagem, expresso em porcentagem, o que pode alcançar o máximo $176,8 \%{ }^{3}$. Quando a pontuação resultante é superior a $100 \%$ significa que foi necessário mais de um profissional para a realização da assistência ${ }^{5}$. Cada ponto do NAS pode ser convertido em 0,24 horas, desse modo, tem-se a informação de quantas horas de trabalho da equipe de enfermagem foram dedicadas a cada paciente ${ }^{6}$.

A carga de trabalho em enfermagem é compreendida como a quantidade de tempo dedicado ao paciente (direta e indiretamente) e ao desenvolvimento profissional ${ }^{7}$. Fatores como o aumento da incidência de infecção hospitalar, a ocorrência de lesões por pressão e de erros relacionados à assistência em saúde, foram descritos como relacionados à carga de trabalho aumentada ${ }^{8}$, sendo que esses fatores podem ocasionar hospitalização prolongada, aumento de custos institucionais e públicos, além de colocar em risco a vida de pacientes e aumentar as taxas de morbimortalidade 2 .

Estudos anteriores sobre a aplicação do $\mathrm{NAS}^{9-11}$ mostraram que esse instrumento é eficiente para estimar o quantitativo de profissionais de enfermagem. Além disso, apontaram ainda lacunas na literatura, sugerindo a necessidade de outros estudos, sobretudo no âmbito específico de unidades neonatais, haja vista que é frequentemente aplicado em Unidade de Terapia Intensiva (UTI) com pacientes adultos.

Considerando que a utilização de instrumentos para avaliação da carga de trabalho permite direcionar o processo de trabalho, esse estudo teve como objetivo analisar a carga de trabalho de enfermagem em uma unidade de internação neonatal e correlacionar às características clínicas dos pacientes.

\section{MÉTOdO}

Trata-se de um estudo descritivo e transversal, realizado em unidade neonatal de referência de um hospital público de ensino, de nível de atenção terciário do Estado de São Paulo, Brasil, que abrange cuidados intensivos e intermediários. Essa unidade conta com 30 leitos, sendo 15 destinados a cuidados intensivos e 15 a intermediários, com taxa média de ocupação de $108 \%$.

A amostra foi do tipo não probabilística, por conveniência. Para fins de dimensionamento de pessoal, recomendase que os pacientes sejam acompanhados por um período mínimo de 30 dias, com a aplicação do NAS uma vez ao dia, para que a amostra possa refletir o perfil dos pacientes atendidos ${ }^{12}$. No presente estudo, optou-se por incluir todos os pacientes da unidade que permaneceram hospitalizados por, no mínimo, 24 horas ao longo de um período de 60 dias, no ano de 2017. Foi utilizado um instrumento de coleta de informações de caracterização dos pacientes, desenvolvido para o presente estudo, contemplando as variáveis: sexo, número de dias de vida, dias de hospitalização, peso ao nascer, idade gestacional ao nascer, classificação quanto à idade gestacional, classificação quanto ao crescimento intrauterino, Apgar, motivo de internação, procedência (centro obstétrico, alojamento conjunto e pronto atendimento) e desfecho da internação no dia da coleta (alta, transferência, óbito, ou se ainda permanecia hospitalizado). O instrumento NAS foi aplicado para avaliar a carga de trabalho de enfermagem ao longo do período estudado, considerando-se sempre as últimas 24 horas do dia anterior à coleta de dados.

A coleta de dados foi realizada por uma das autoras e por duas auxiliares de pesquisa, que foram previamente preparadas para coleta por meio de um treinamento com duração de quatro horas. Para assegurar a uniformidade da avaliação, foram aplicados os instrumentos de coleta de dados por três dias consecutivos no mesmo paciente. Também foi utilizado conteúdo atualizado sobre o $\mathrm{NAS}^{13}$, o que uniformizou o significado de cada um de seus itens.

Os dados tabulados em planilha eletrônica foram submetidos a análise descritiva e inferencial. A distribuição quanto à normalidade foi avaliada por meio do teste de Kolmogorov-Smirnov. Foi investigada a correlação entre a carga de trabalho (pontuação NAS) e variáveis como dias de vida, peso de nascimento e idade gestacional. Essas correlações foram testadas por meio do coeficiente de correlação de Spearman, coeficiente não-paramétrico e que varia de -1 a 1. Para interpretação dos resultados dos coeficientes de correlação considerou-se 0,1 a 0,29 (correlação fraca), 0,30 a 0,49 (correlação moderada) e maior ou igual a 0,50 (correlação forte) $)^{14}$.

Para as comparações envolvendo uma variável qualitativa com duas categorias e uma variável quantitativa foi aplicado o teste não-paramétrico de Mann-Whitney ou o teste t de Student não pareado. Foi calculada a variação da pontuação NAS ao longo dos dias de avaliação, além da pontuação média do NAS para identificar os recém-nascidos que mantinham pontuação estável, ou seja, que não apresentavam variação relevante da carga de trabalho. Para as análises foram utilizados os softwares Statistical Analysis System (SAS), versão 9.4, e Statistical Package for the Social Sciences (SPSS), versão 22. A pesquisa foi aprovada pelo Comitê de Ética em Pesquisa, sob número de protocolo 2.238328/2017 (CAAE 73058317800005404). 


\section{RESULTADOS E DISCUSSÃo}

A amostra foi constituída por 115 pacientes, a maioria dos pacientes pertencia ao sexo masculino, corroborando com outros estudos ${ }^{1,10,11}$. Quanto a idade gestacional, 72,2 \% eram prematuros, com média de 35 semanas, variando de 24 a 41 semanas. Esse perfil é justificado por se tratar de uma unidade terciária, com atendimento regionalizado, o que difere do perfil de recém-nascidos de estudo anterior, em que se observou maior frequência de bebês nascidos a termo ${ }^{2}$. Os prematuros são aqueles que apresentam maiores chances de instabilidade clínica e, consequentemente, maior número de intervenções terapêuticas.

Considerando o desfecho dos pacientes, houve maior frequência de alta ( $n=61,53 \%)$, seguida dos que permaneceram internados até o fim da coleta de dados $(n=32,27,8 \%)$. A pontuação média do NAS apresentou valor elevado (73\%), sendo que na unidade de cuidados intensivos esta pontuação foi de $74 \%$ e na unidade de cuidados semiintensivos 64\%; valores superiores ao identificado em estudo anterior ${ }^{11}$. Em outro estudo recente a pontuação média do NAS foi de 48,5 a 50\% para pacientes cuja complexidade de cuidados era semi-intensiva, ou seja, quando comparados aos do presente estudo, apresentaram valores inferiores ${ }^{10}$. Ressalta-se que a pontuação média do NAS variou de 41 a $87 \%$ em estudos que abordaram outras especialidades ${ }^{5,6,15-17}$

Considera-se como pontuações altas do NAS valores superiores a 50\%: nesse contexto um profissional de enfermagem teria disponibilidade de cuidar integralmente apenas de um paciente por turno de trabalho ${ }^{9}$, sendo inviável a proporção preconizada pela legislação de um técnico de Enfermagem cada dois pacientes ${ }^{18}$. Identificou-se, portanto, a necessidade de revisão das legislações quanto à recomendação dos valores de proporção do número de profissionais por leito.

Ao analisar as correlações entre a variação da pontuação do NAS e as variáveis peso de nascimento $(p<0,001)$ e idade gestacional $(p<0,001)$, a carga de trabalho foi inversamente proporcional. Assim, quanto maior a idade gestacional e o peso, menor a variação da pontuação do NAS, o que corrobora com achado na literatura ${ }^{16}$, ou seja, recém-nascidos com maior idade gestacional demandam menor carga de trabalho de enfermagem. Embora tenha sido identificada fraca correlação, verificou-se também que quanto maior o número de dias de vida ou dias de hospitalização, maior a pontuação média do $\mathrm{NAS}^{16}$, o que pode estar relacionado aos pacientes crônicos e sob cuidados paliativos. Para dimensionar adequadamente a equipe de enfermagem e proporcionar dessa forma uma assistência segura, as unidades neonatais devem conhecer o perfil da clientela atendida.

A Tabela 1 apresenta a frequência das atividades de enfermagem. Foram efetuadas, no total, 1944 aplicações do NAS. Foi realizada a somatória das pontuações para obtenção da pontuação NAS diária da unidade. A menor pontuação média NAS por dia foi de $58 \%$ e a maior $98 \%$, enquanto a média geral da unidade diária foi de $73 \%$. Verificou-se, portanto, que os recém-nascidos necessitaram em média de 17,5 horas de assistência de enfermagem, com um mínimo de 14 horas e máximo de 23,5 horas, dentro das 24 horas do dia.

$\mathrm{Na}$ unidade de cuidados semi-intensivos, a carga de trabalho obteve pontuação média de $64 \%$, enquanto na de cuidados intensivos essa carga foi de $74 \%$. Obteve-se maior frequência de aplicações na unidade de cuidados semi intensivos $(57,71 \%)$, porém a unidade intensiva apresentou a maior pontuação, no entanto.

Em ordem decrescente, os itens terapêuticos com maior frequência nas aplicações foram: 1b (presença à beira do leito por duas horas ou mais em algum plantão); 3 (medicação, exceto drogas vasoativas); 21 (alimentação enteral sonda nasoenteral, jejunostomia) e 7a (suporte e cuidado com a família e paciente que requerem dedicação exclusiva por uma hora em algum plantão). Tais resultados são justificados por se tratar de um perfil de clientes que dependem integralmente da enfermagem e/ou de familiares para realização dos cuidados. Houve uma frequência elevada dos itens relativos às atividades de suporte e cuidado com a família, com percentual de 8,2\% para três horas ou mais e de $64 \%$ por pelo menos uma hora, mostrando que é despendido tempo significativo nestas atividades.

É interessante ressaltar que o item relacionado a mobilização e posicionamento com frequência superior a três vezes em 24 horas ou com dois enfermeiros foi apontado por $100 \%$ dos profissionais de enfermagem da equipe. Outras atividades que apresentaram maior frequência de relato pelos profissionais foram os itens de monitorização e controles à beira do leito por duas horas ou mais, medicação e alimentação enteral (sonda nasoenteral, jejunostomia).

O item 15 do NAS, que diz respeito ao atendimento em parada cardiorrespiratória, obteve menor frequência de pontuação, possivelmente por se tratar de uma unidade com alta tecnologia e que conta com preparo de profissionais para o reconhecimento de sinais de deterioração da condição clínica do neonato e que, portanto, podem antecipar medidas que previnam a ocorrência desse evento. Além disso, tem-se a própria peculiaridade dessa clientela de recuperar-se mais prontamente aos eventos que desencadeiam a parada cardiorrespiratória ${ }^{19}$. 
TABELA 1: Frequência dos itens terapêuticos do Nursing Activities Score ( $n=1944$ medidas). Campinas, SP, Brasil, 2017.

\begin{tabular}{|c|c|c|}
\hline Atividades do Nursing Activities Score & $\mathbf{n}$ & $\%$ \\
\hline \multicolumn{3}{|l|}{ 1. Monitorização e controles } \\
\hline 1a. SSVV e balanço hídrico & 260 & 13,37 \\
\hline 1b. beira do leito por duas horas ou mais & 1682 & 86,52 \\
\hline 1c. beira do leito por quatro horas ou mais & 0 & 0 \\
\hline 2. Investigações laboratoriais & 978 & 50,30 \\
\hline 3. Medicação, exceto drogas vasoativas & 1600 & 82,30 \\
\hline \multicolumn{3}{|l|}{ 4. Procedimentos de higiene } \\
\hline 4a. procedimentos de higiene (descrição dos habituais) & 956 & 49,17 \\
\hline 4b. procedimentos de higiene por mais de duas horas & 985 & 50,66 \\
\hline 4c. procedimentos de higiene por mais de quatro horas & 0 & 0 \\
\hline 5. Cuidados com drenos (exceto sonda nasogástrica) & 10 & 0,51 \\
\hline \multicolumn{3}{|l|}{ 6. Mobilização e posicionamento } \\
\hline 6a. realização até três vezes em 24 horas & 0 & 0 \\
\hline 6b. realização mais do que três vezes em 24 horas ou com dois enfermeiros & 1944 & 100 \\
\hline 6c. realização com três enfermeiros ou mais & 0 & 0 \\
\hline \multicolumn{3}{|l|}{ 7. Suporte e cuidado à família e paciente } \\
\hline 7a. suporte e cuidado com a família e paciente por uma hora & 1244 & 63,99 \\
\hline 7b. suporte e cuidado com a família e paciente por três horas ou mais & 160 & 8,23 \\
\hline \multicolumn{3}{|l|}{ 8. Tarefas administrativas e gerenciais } \\
\hline 8a. tarefas de rotina & 1156 & 59,46 \\
\hline 8b. tarefas administrativas e gerenciais por duas horas & 127 & 6,53 \\
\hline 8c. tarefas administrativas e gerenciais por quatro horas ou mais & 0 & 0 \\
\hline 9. Suporte respiratório & 796 & 40,94 \\
\hline 10.Cuidados com vias aéreas artificiais, tubo orotraqueal ou traqueostomia & 267 & 13,73 \\
\hline 11. Tratamento para melhora de função pulmonar & 1087 & 56,00 \\
\hline 12.Medicação vasoativa & 55 & 2,82 \\
\hline 13.Reposição volêmica com mais de $3 \mathrm{l} / \mathrm{m}^{2} /$ dia & 25 & 1,28 \\
\hline 14.Monitorização átrio esquerdo. Cateter artéria pulmonar & 0 & 0 \\
\hline 15.Reanimação cardiorrespiratória, exceto soco precordial & 4 & 0,20 \\
\hline 16. Hemofiltração. Técnicas dialíticas & 0 & 0 \\
\hline 17.Medida quantitativa do débito urinário & 922 & 47,42 \\
\hline 18.Medida da pressão intracraniana & 0 & 0 \\
\hline 19.Tratamento acidose/alcalose metabólica complicada & 10 & 0,51 \\
\hline 20.Hiperalimentação intravenosa & 342 & 17,59 \\
\hline 21.Alimentação enteral (sonda nasoenteral, jejunostomia) & 1518 & 78,08 \\
\hline 22.Intervenções específicas na unidade de terapia intensiva & 304 & 15,63 \\
\hline 23.Intervenções específicas fora da Unidade & 23 & 1,18 \\
\hline
\end{tabular}

Como se observa na Tabela 1, alguns itens não foram pontuados por não serem observados comumente em unidade neonatal, bem como não ocorreram alguns procedimentos específicos no período de coleta de dados da pesquisa, como utilização de técnicas dialíticas, por exemplo.

A Tabela 2 apresenta resultados do teste de correlação de Spearman para as correlações entre as variáveis quantitativas (dias de vida, dias de hospitalização, peso de nascimento, idade gestacional) e a pontuação NAS, considerando a pontuação média do NAS e a variação da pontuação do NAS.

Embora haja uma organização de estrutura física com número específico de leitos para Cuidados Semi-Intensivos e para cuidados intensivos, onde o estudo foi realizado, há três leitos da área de Semi-Intensivos que costumam sempre estar ocupados por pacientes intensivos, devido à demanda dessa classificação de pacientes ser superior ao número de leitos atual da área de UTI desta unidade. É importante destacar que, geralmente, o número de pacientes neonatais classificados como intensivos e alocados na Unidade de Semi-intensivos supera esses três leitos previstos. Esse contexto justifica o fato de que $21 \%$ das 1122 medidas realizadas na Unidade de Semi-intensivos foram consideradas de pacientes intensivos, o que supera em $5 \%$ os valores esperados para os três leitos estimados. Verificou-se que não houve ampla variação da pontuação média do NAS dos pacientes sob cuidados semi-intensivos quando comparados aos intensivos.

É essencial considerarmos que a sobrecarga de trabalho é o principal fator causador da síndrome de Burnout na equipe de enfermagem ${ }^{20,21}$, associada ao aumento do absenteísmo, maiores chances de abandono da área de enfermagem, alta rotatividade da equipe, redução da qualidade da assistência, aumento do tempo de internação dos pacientes, bem como aumento dos erros relacionados à assistência e, portanto, prejuízos à segurança do paciente ${ }^{22}$. 
TABELA 2: Correlação entre as características clínicas-demográficas e carga de trabalho ( $n=115)$. Campinas, SP, Brasil, 2017.

\begin{tabular}{|c|c|c|}
\hline Características clínico-demográficas & NAS - média & NAS - variação \\
\hline \multirow[t]{3}{*}{ Dias de vida } & $0,2014^{*}$ & $0,0926^{*}$ \\
\hline & $0,0309 * *$ & $0,3274 * *$ \\
\hline & 115 & $114^{+}$ \\
\hline \multirow[t]{3}{*}{ Dias de hospitalização } & $0,2057^{*}$ & $0,1326^{*}$ \\
\hline & $0,0274 * *$ & $0,1595^{* *}$ \\
\hline & 115 & $114^{+}$ \\
\hline \multirow[t]{3}{*}{ Peso de nascimento } & $-0,1767^{*}$ & $-0,4236^{*}$ \\
\hline & $0,0589 * *$ & $<0,0001 * *$ \\
\hline & 115 & $114^{\dagger}$ \\
\hline \multirow[t]{3}{*}{ Idade gestacional } & $-0,2580^{*}$ & $-0,4500^{*}$ \\
\hline & $0,0054 * *$ & $<0,0001 * *$ \\
\hline & 115 & $114^{\dagger}$ \\
\hline
\end{tabular}

Os resultados obtidos no presente estudo com o NAS mostraram-se coerentes com a realidade enfrentada em relação à carga de trabalho da equipe de enfermagem, ademais, recomenda-se para estudos futuros avaliar a correlação desta com os indicadores relativos à segurança do paciente. Sugere-se ainda adaptação de itens do NAS, de forma que possam ser mais fidedignos aos pacientes em unidades neonatais de internação.

\section{Limitações do estudo}

Como uma limitação desse estudo, considera-se a impossibilidade de mensurar aspectos específicos da carga de trabalho no contexto de uma instituição hospitalar de ensino, uma vez que estão presentes nas atividades assistenciais graduandos das áreas de enfermagem, medicina, fisioterapia, fonoaudiologia e residentes da área médica e multiprofissional: característica que difere de outros hospitais e que pode representar um incremento à carga de trabalho profissional. Unidade essa que apresenta elevada carga de trabalho, segundo estudo realizado com aplicação do Nursing Activities Score (NAS) ${ }^{23}$.

\section{CONCLUSÃO}

Foi identificada elevada carga de trabalho de enfermagem na unidade neonatal estudada, que apresentou pontuação NAS de $73 \%$, ou seja, nessa unidade dedica-se uma média de 17,5 horas de assistência direta a cada paciente nas 24 horas de cuidado.

Para dimensionar adequadamente a equipe de enfermagem é importante conhecer o perfil da clientela atendida. $\mathrm{Na}$ amostra estudada, verificou-se que quanto menor a idade gestacional e o peso, maior foi a carga de trabalho. $\mathrm{O}$ número maior de dias de vida do recém-nascido e de dias de hospitalização na unidade também podem significar incremento na carga de trabalho dos profissionais. A investigação sobre a carga de trabalho tem como objetivo gerenciar fatores que promovam a segurança do paciente e de seus familiares atendidos, bem como subsidiar dados para o adequado dimensionamento de pessoal de enfermagem em unidades neonatais, o que favorecerá boas condições de trabalho e satisfação.

\section{REFERÊNCIAS}

1. Grebinski ATKG, Biederman FA, Berte C, Barreto GMS, Oliveira JLC, Santos EB. Workload and sizing of therapy in nursing staff intensive newborn. Enferm. Foco [Internet]. 2019 [cited 2020 Nov 30]; 10(1):24-8. Available from:

http://biblioteca.cofen.gov.br/wp-content/uploads/2019/02/Carga-De-Trabalho-e-Dimensionamento-De-Pessoal-DeEnfermagem-Em-Terapia-Intensiva-Neonatal.pdf.

2. Françolin L, Gabriel CS, Bernardes A, Silva AEBC, Brito MFP, Machado JP. Patient safety management from the perspective of nurses. Rev. Esc. Enferm. USP [Internet]. 2015 [cited 2017 Oct 12]; 49(2):277-83. DOI: http://dx.doi.org/10.1590/S0080623420150000200013.

3. Miranda DR, Nap R, Rijk A, Schaufeli W, lapichino G. Nursing Activities Score. Crit. care med. [Internet]. 2003 [cited 2018 Sep 10]; 31(2):374-82. DOI: http:///dx.doi.org/10.1097/01.ccm.0000045567.78801.cc.

4. Queijo AF, Padilha KG. Nursing Activities Score (NAS): Cross-cultural adaptation and validation to Portuguese language. Rev. Esc. Enferm. USP [Internet]. 2009 [cited 2017 Nov 10]; 43(Esp):1018-25. Available from: https://www.revistas.usp.br/reeusp/article/view/40418. 
5. Oliveira EM, Secco LMD, Figueiredo WB, Padilha KG, Secoli SR. Nursing Activities Score and the cost of nursing care required and available. Rev. Bras. Enferm. [Internet]. 2019 [cited 2020 Oct 21]; 72(Suppl 1):137-42. DOI: http://dx.doi.org/10.1590/00347167-2017-0655

6. Amadeu LM, Dell'Acqua MCQ, Castro MCN, Palhares VC, Serafim CTR, Trettene AS. Nursing workload in burn intensive care unit. Rev. Bras. Enferm. [Internet]. 2020 [cited 2020 Nov 30]; 73(Suppl 1):e20190446. DOI: http://dx.doi.org/10.1590/00347167-2019-0446

7. Alghamdi MG. Nursing workload: a concept analysis. J. nurs. Manag. [Internet]. 2016 [cited 2018 Nov 30]; 24(4):449-7. DOI: http://dx.doi.org/10.1111/jonm.12354.

8. Magalhães AMM, Costa DG, Riboldi CO, Mergen T, Barbosa AS, Moura GMSS. Association between workload of the nursing staff and patient safety outcomes. Rev. Esc. Enferm. USP [Internet]. 2017 [cited 2019 Sep 20]; 51:e03255. DOI: http://dx.doi.org/10.1590/S1980-220X2016021203255.

9. Souza VS, Inoue KC, Oliveira JLC, Magalhães AMM, Martins EAP, Matsuda LM. Sizing of the nursing staff in adult intensive therapy. REME - Rev. Min. Enferm. [Internet]. 2018 [cited 2019 Nov 21]; 22:e-1121. DOI: http://dx.doi.org/10.5935/14152762.20180056.

10. Branco LLWV, Beleza LO, Luna AA. Nursing workload in neonatal ICU: application of the nursing activities score tool. J. res.: fundam. care. online [Internet]. 2017 [cited 2019 Feb 19]; 9(1):144-51. DOI: http://dx.doi.org/10.9789/21755361.2017.v9i1.144-151

11. Maziero ECS, Cruz EDA, Alpendre FT, Brandão MB, Teixeira FFR, Krainski ET. Association between nursing work conditions and adverse events in neonatal and pediatric Intensive Care Units. Rev. Esc. Enferm. USP [Internet]. 2020 [cited 2020 Nov 17]; 54:e03623.DOI: https://doi.org/10.1590/S1980-220X2019017203623.

12. Fugulin FMT, Gaidzinski RR, Castilho V. Dimensionamento de pessoal de enfermagem em instituições de saúde. In: Kurcgant $P$. Gerenciamento em enfermagem. 3.ed. Rio de Janeiro: Guanabara Koogan; 2016. p.115-27.

13. Padilha KG, Stafseth S, Solms D, Hoogendoom M, Monge FJ, Miranda DR, et. al. Nursing Activities Score: an updated guideline for its application in the Intensive Care Unit. Rev. Esc. Enferm. USP [Internet]. 2015 [cited 2020 Feb 18]; 49(Esp):131-7. DOI: http://dx.doi.org/10.1590/S0080-623420150000700019.

14. Cohen J. Statistical power analysis for the behavioral sciences. 2a ed. New Jersey:

15. Nassiff A, Araújo TR, Meneguetti MG, Rodrigues FB, Filho AB, Laus AM. Nursing workload and patient mortality at an intensive care unit. Texto \& contexto enferm. [Internet]. 2018 [cited 2019 Nov 21]; 27(4):1-7. DOI: http://dx.doi.org/10.1590/010407072018000390017.

16. Salgado PO, Januário CF, Toledo LV, Brinati LM, Araújo TS, Boscarol GT. Nursing workload required by patients during ICU admission: a cohort study. Enferm. glob. [Internet]. 2020 [cited 2020 Dec 01]; 19(59):450-78.DOI: http://doi.org/10.6018/eglobal.400781.

17. Lucchini A, Elli S, Felippis C, De Fellippis C, Greco C, Mulas A, Ricucci P, et al., The evaluation of nursing workload within an Italian ECMO Centre: A retrospective observational study. Intensive \& Critical Care Nursing [Internet]. 2019 [cited 2020 Dec 02]. 55. DOI: https://doi.org/10.1016/j.iccn.2019.07.008.

18. Conselho Federal de Enfermagem. Resolução COFEN n. 543, de 18 de abril de 2017. Atualiza e estabelece parâmetros para o Dimensionamento do Quadro de Profissionais de Enfermagem nos serviços/locais em que são realizadas atividades de enfermagem [Internet]. Brasília: COFEN; 2017 [cited 2018 Mar 14]. Available from: http://www.cofen.gov.br/resolucao-cofen5432017_51440.html.

19. Bernoche C, Timerman S, Polastri TF, Giannetti NS, Siqueira AWS, Piscopo A, et. al., Atualização da Diretriz de Ressuscitação Cardiopulmonar e Cuidados Cardiovasculares de Emergência da Sociedade Brasileira de Cardiologia Arq. Bras. Cardiol. [Internet]. 2019 [cited 2019 Nov 06]; 113(3):449-663. DOI: https://doi.org/10.5935/abc.20190203.

20. Rodrigues CCFM, Santos VEP, Sousa P. Patient safety and nursing: interface with stress and Burnout Syndrome. Rev Bras Enferm [Internet]. 2017 [cited 2020 Mar 15]; 70(5):1083-8. DOI: http://dx.doi.org/10.1590/0034-7167-2016-0194.

21. Andolhe R, Barbosa RL, Oliveira EM, Costa ALS, Padilha KG. Stress, coping and burnout among Intensive Care Unit nursing staff: associated factors. Rev. Esc. Enferm. USP [Internet]. 2015 [cited 2020 Oct 19]; 49(Esp):57-63. DOI: https://doi.org/10.1590/S0080-623420150000700009.

22. Carvalho DP, Rocha LP, Pinho EC, Tomaschewski-Barlem JG, Barlem ELD, Goulart LS. Workloads and burnout of nursing workers. Rev. Bras. Enferm. [Internet]. 2019 [cited 2020 Nov 05]; 72(6):1435-41. DOI: http://dx.doi.org/10.1590/0034-7167-2017-0659.

23. Dini AP, Oliveira ACV, Almeida-Hamasaki BP, Quinteiro NM, Carmona EV. Adaptation of an instrument to classify neonatal patients into care categories. Rev. Esc. Enferm. USP [Internet]. 2021 [cited 2021 Apr 19]; 55:e03674. DOI: http://dx.doi.org/10.1590/s1980-220x2019033603674 